\title{
La carga inmediata con implantes Microdent en el maxilar superior.I. Aspectos quirúrgicos
}

\author{
VELASCO ORTEGA E* \\ FORNÉS ORTUÑO E** \\ GARCÍA MÉNDEZ A*** \\ MEDEL SOTERAS $\mathbf{R} * * *$ \\ LÓPEZ FRÍAS $\mathrm{J} * * * *$
}

Velasco Ortega E, Fornés Ortuño E, García Méndez A, Medel Soteras R, López Frías J. La carga inmediata con implantes Microdent en el maxilar superior. I. Aspectos quirúrgicos. Av Periodon Implantol. 2007; 19, Suppl.: 9-16.

\section{RESUIMIEN}

Introducción. El objetivo del presente trabajo era valorar el protocolo quirúrgico de la carga inmediata en el tratamiento con implantes oseointegrados del maxilar superior.

La carga inmediata de los implantes orales para la rehabilitación del maxilar superior constituye una evaluación integral que incluye una valoración preoperatoria. Antes de la cirugía, las condiciones sistémicas y orales, así como el diagnóstico radiográfico (ortopantomografía y TAC) son obtenidos. La prevención antimicrobiana se realiza con antibióticos (amoxicilina y clavulánico) y clorhexidina. La anestesia local se consigue con articaína/adrenalina.

La cirugía comienza con una incisión supracrestal o mediante la técnica sin colgajo. Los implantes con prótesis solidaria pueden ser insertados según una distribución anteroposterior mediante una guía quirúrgica. Una apropiada técnica quirúrgica y un buen diseño del implante con superficie rugosa mejora la estabilidad primaria y la oseointegración. El número y tamaño de los implantes es seleccionado de acuerdo al diagnostico y a su valoración protésica.

Conclusiones. Este estudio indica que el protocolo quirúrgico del tratamiento del maxilar superior mediante la carga inmediata de los implantes puede constituir una terapéutica exitosa.

\section{PALABRAS CLAVE}

Implantes orales, carga inmediata, prótesis fija, implantología oral, maxilar superior.

Fecha de recepción: Septiembre 2006.

Aceptado para publicación: Septiembre 2006. 


\section{INTRODUCCIÓN}

En la actualidad, el tratamiento del paciente edéntulo con implantes oseointegrados representa una alternativa con éxito al tratamiento convencional con prótesis completa removible. Desde los protocolos clásicos implantológicos, las diversas fases quirúrgicas y prostodóncicas han ido evolucionando con la incorporación de técnicas como la cirugía sin realización de colgajo y la carga precoz e inmediata ${ }^{1-3}$.

Los nuevos protocolos de carga precoz e inmediata han acortado el tiempo de espera de los pacientes y han mejorado notablemente su calidad de vida, transformando su estado edéntulo parcial o total en un estado dentado con una repercusiones funcionales, estéticas y psicológicas muy favorables ${ }^{4}$. La carga inmediata ha sido utilizada y evaluada positivamente en la rehabilitación del paciente con edentulismo total mandibular mediante la realización de sobredentaduras y prótesis fija (prótesis inmediata híbrida atornillada provisional y prótesis fija definitiva a los 3 meses) con resultados satisfactorios ${ }^{5-9}$.

Con respecto al maxilar superior, parece que la supervivencia de los implantes cargados de forma inmediata con prótesis fija es similar a los implantes cargados de forma convencional. Sin embargo no existen muchos estudios al respecto con un número importante de pacientes ${ }^{3}$. Durante un periodo de 5 años, Degidi y Piatelli ${ }^{10}$ trataron 152 pacientes con $646 \mathrm{im}-$ plantes en casos de edentulismo parcial y total, de los que 14 pacientes fueron tratados con 133 implantes con carga inmediata en el maxilar superior. El periodo de seguimiento clínico fue de 2 a 60 meses. 2 implantes se perdieron lo que indica una supervivencia del $98 ; 5 \%$. No hubo ningún problema prostodóncico ${ }^{10}$. Este estudio ha continuado y recientemente se ha publicado los resultados clínicos a 5 años de 388 implantes insertados en el maxilar superior de 43 pacientes (media de 9 implantes) con un éxito del $98 \%{ }^{11}$. Un estudio argentino reciente realizado sobre 26 pacientes y 217 implantes con carga inmediata en maxilares edéntulos ( 6 pacientes con prótesis de resina, 8 pacientes con prótesis de resina reforzadas con metal y 12 con prótesis definitiva) seguidas durante un periodo de 12 a 74 meses refiere un éxito del $100 \%{ }^{12}$.

\section{DIAGNÓSTICO IMIPLANTOLÓGICO}

La carga inmediata de los implantes en el maxilar superior exige un diagnóstico previo donde se valore de forma precisa los aspectos generales y orales que pueden condicionar los resultados a corto, medio y largo plazo del tratamiento implantológico. Los pacientes que padezcan trastornos sistémicos graves que pueden comprometer la oseointegración deben ser excluidos de los protocolos de carga inmediata y ser tratados con técnicas de implantología oral convencional o con otras alternativas prostodóncicas (prótesis removible o fija). En este sentido, los pacientes con trastornos metabólicos (ej. osteoporosis, diabetes) que puedan interferir en la cicatrización de los implantes - afectar al hueso maxilar deben ser evitados. Así mismo los pacientes irradiados o fumadores pueden presentar unas menores expectativas de éxito con los protocolos de carga inmediata y deben ser considerados como pacientes de alto riesgo ${ }^{13-14}$.

Desde un punto de vista oral, todos los pacientes que van a ser tratados con carga inmediata en el maxilar superior deben ser informados del protocolo del tratamiento con implantes, de los aspectos quirúrgicos y prostodóncicos, de la temporalización del tratamiento y del seguimiento clínico, así como de la posibilidad de la existencia de complicaciones y pérdida de implantes. Todos los pacientes deben autorizar el tratamiento implantológico mediante un consentimiento informado.

Antes de la realización del tratamiento implantológico con carga inmediata, todos los pacientes deben ser valorados radiológicamente con una ortopantomografía. Cuando las características clínicas y anatómicas del paciente lo requieran (frecuentemente) se realizará una tomografía axial computarizada (TAC) que permitirá una valoración real del tamaño, estructura, y disposición del maxilar en un sentido transversal y longitudinal mediante los sucesivos cortes realizados. Así mismo, las nuevas técnicas tridimensionales de imagen asistidas por ordenador pueden ser útiles al configurar la posible localización de los implantes teniendo en cuenta las características morfológicas del maxilar superior ${ }^{15}$.

Posteriormente, es necesario la toma de impresiones y el desarrollo de unos modelos de estudio montados en un articulador con las diferentes posibilidades prostodóncicas funcionales y estéticas y su relación con la disposición y número de implantes. Así mismo, es necesario valorar la arcada antagonista mandibular si es dentada (parcial o total) o edéntula (parcial o total) y en este último caso el tipo de prótesis que lleva el paciente (removible o fija) que puede condicionar el número de implantes necesarios y el diseño de la prótesis definitiva superior ${ }^{16}$. 


\section{TÉCNICA QUIRÚRGICA}

La inserción de varios implantes en el maxilar superior para realizar una carga inmediata supone la integración en un solo tiempo quirúrgico (Figuras 1-6) de todos los procedimientos clínicos necesarios para la rehabilitación inmediata con una prótesis fija (generalmente provisional). Esta modalidad terapéutica implantológica exige que el profesional tenga la experiencia clínica suficiente para abordar los diferentes tiempos (ej. extracciones de dientes naturales, inserción de implantes) y técnicas quirúrgicas (ej. osteótomos, elevación sinusal) de forma secuenciada ${ }^{13}$.

Previamente a la cirugía (2-3 horas antes), los pacientes deben comenzar un régimen antibiótico preventivo (amoxicilina + clavulánico) durante una semana. Así mismo, los pacientes deben ser tratados durante una semana con ibuprofeno. A los pacientes se les realiza anestesia local. A la semana se retiran las suturas, excepto en los casos de cirugía sin colgajo. A todos los pacientes se les recomienda el enjuague o aplicación con spray diario con clorhexidina durante los primeros 30 días.

La técnica quirúrgica debe ser lo menos traumática posible ya que un excesivo trauma puede provocar una osteonecrosis y una encapsulación fibrosa del implante. El fresado a velocidad reducida (menos de 800 r.p.m) para evitar el sobrecalentamiento del lecho implantario es recomendable ${ }^{13}$.

Es recomendable la realización de una guía quirúrgica que represente la futura disposición de la prótesis dental para intentar insertar los implantes de la forma más adecuada con el objetivo de que se correspondan posteriormente con la emergencia de los dientes protéticos (Figuras 1-6) ${ }^{16}$.

Durante el desarrollo de la implantología oral, un requisito clásico para obtener la oseointegración con éxito era la inserción sumergida de los implantes (una primera fase quirúrgica) y posteriormente después del tiempo de cicatrización libre de carga funcional (3-6 meses), la realización de una segunda fase quirúrgica con la sustitución de los tornillos de cierre y la colocación de los tornillos de cicatrización con la exposición al medio oral del extremo superior de los implantes ${ }^{1}$. Sin embargo, otros investigadores y clínicos desarrollaron los protocolos del tratamiento con implantes en una sola fase quirúrgica ${ }^{2}$. En este sentido, los actuales protocolos de carga inmediata han modificado notablemente la técnica quirúrgica sumergida y han con-

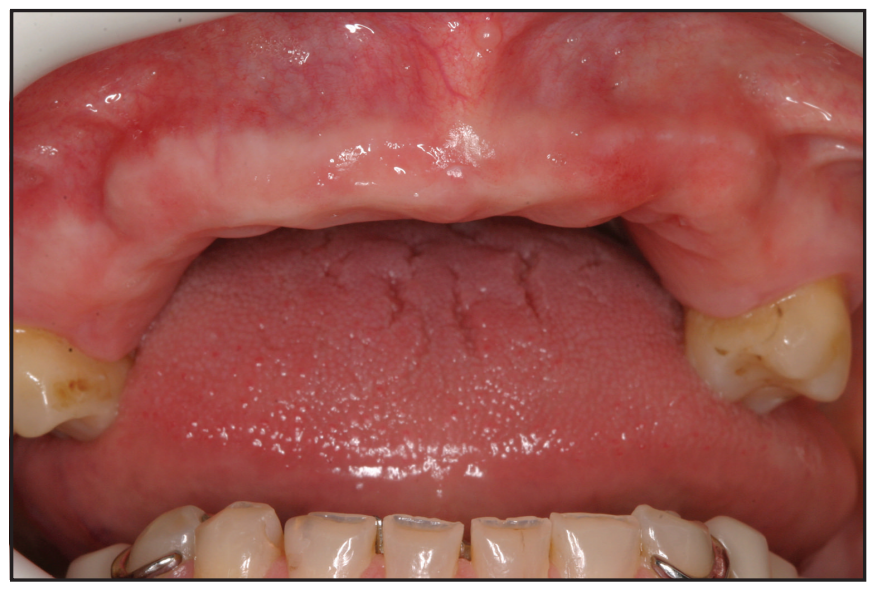

Fig. 1: Aspecto clínico del paciente con el espacio edéntulo maxilar.

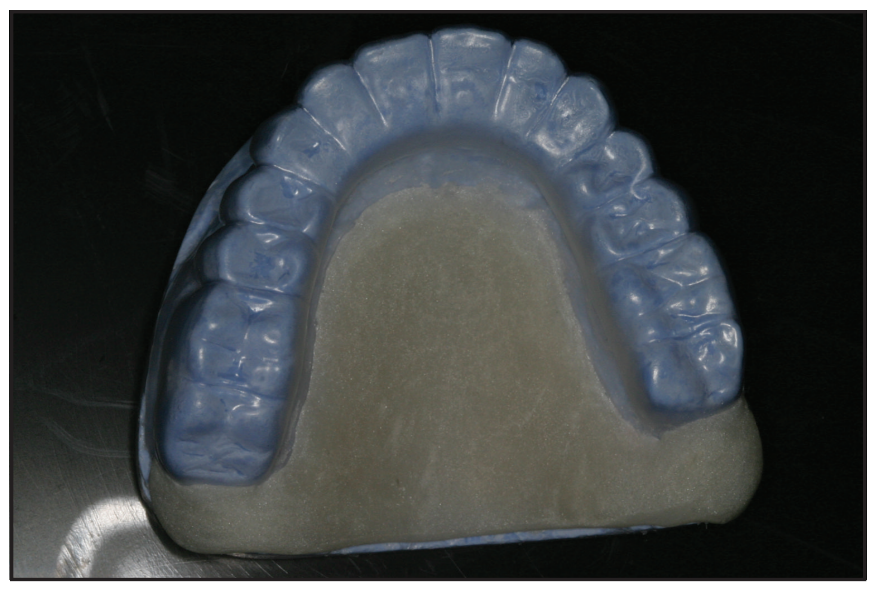

Fig. 2: Realización de una férula quirúrgica con la prótesis removible del paciente.

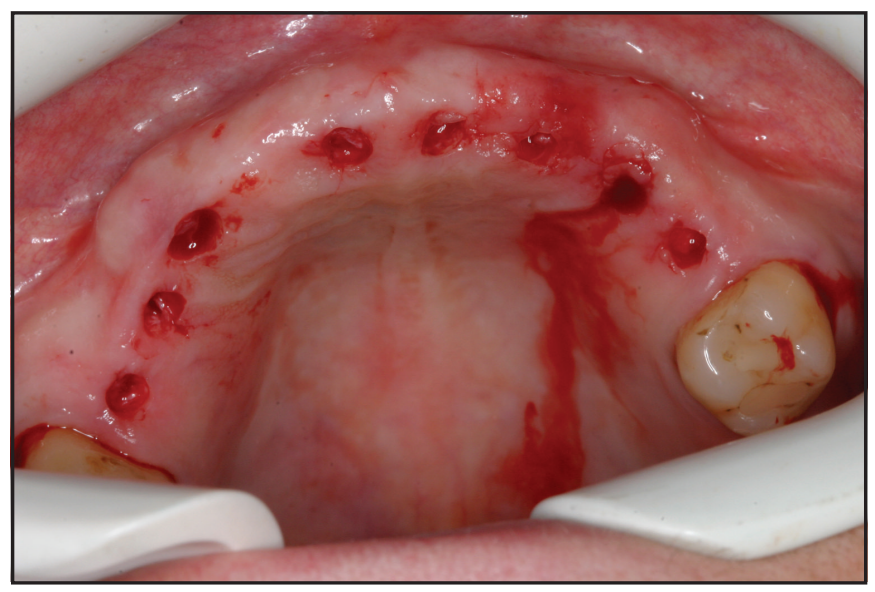

Fig. 3: Señalización de la posición de los implantes. 


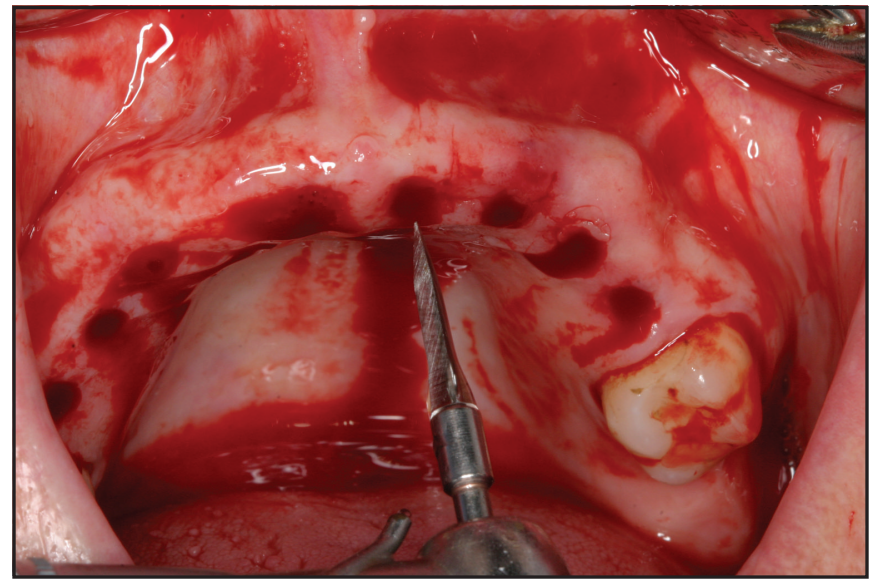

Fig. 4: Cirugía sin colgajo y fresado del hueso maxilar para la inserción de los implantes.

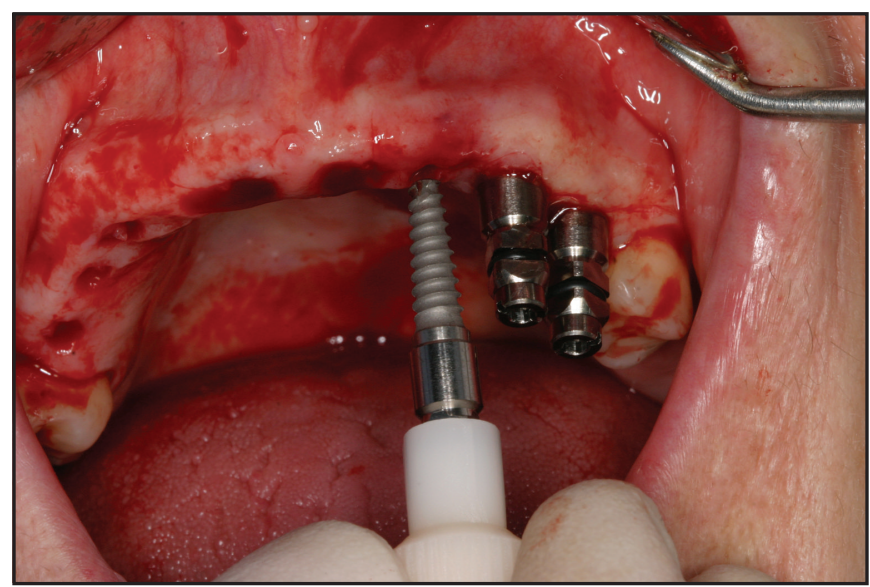

Fig. 5: Inserción de los implantes de una pieza (MRT, Microdent). Se observa los transportadores de los implantes.

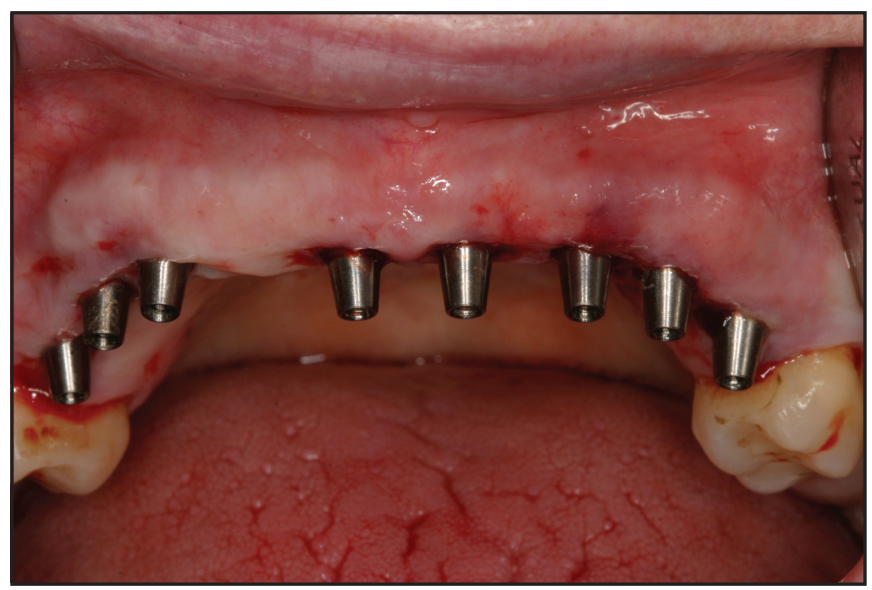

Fig. 6: Aspecto clínico de los implantes MRT insertados con su unión protésica solidaria. firmado los excelentes resultados del tratamiento implantológico no sumergido.

Más recientemente, la técnica sin colgajo se ha ido incorporando a la práctica implantológica en aquellos casos en que los rebordes alveolares residuales no ofrecen problemas de volumen y de morfología que justifiquen la necesidad de realizar una incisión supracrestal convencional. La ventaja fundamental de esta técnica es el postoperatorio excelente y el buen sellado de los tejidos blandos periimplantarios, aunque exige una buena planificación diagnóstica del tejido óseo subyacente y de la disposición tridimensional de los implantes (Figuras 1-6).

\section{ELHUESO MAXILAR}

La carga inmediata del maxilar supone un reto importante en el tratamiento con implantes ya que la densidad del hueso representa un papel esencial para conseguir la oseointegración y determinar el éxito a largo plazo de los implantes. En primer lugar, porque la carga inmediata cuestiona el tiempo convencional de cicatrización de 3-6 meses sin carga. El procedimiento quirúrgico origina un fenómeno de aceleración de la reparación ósea alrededor en la interfase del implante. El hueso laminar organizado y mineralizado se transforma en un entramado óseo de reparación menos mineralizado. La interfase hueso-implante es más débil y presenta un riego mayor entre las 3-6 semanas después de la carga inmediata ${ }^{17}$.

En segundo lugar, desde un punto de vista funcional, el hueso sometido a carga se deforma. Si la carga es fisiológica existe una adaptación que permite su remodelación y permanece como un hueso laminar organizado y mineralizado. Si la sobrecarga es mayor, el hueso el hueso comienza a desaparecer y se forma tejido fibroso. La carga inmediata debe ejercer sobre el hueso maxilar unas fuerzas controladas que se sitúen dentro del margen de respuesta fisiológica para mantener un buen nivel óseo reparativo ${ }^{17}$.

Finalmente, un implante insertado en hueso compacto asegura su estabilidad primaria y favorece la aplicación de las fuerzas que caracterizan la carga funcional inmediata. Sin embargo, en el maxilar superior donde la densidad ósea es menor y la porosidad es más frecuente puede ocasionar un mayor índice de fracasos ${ }^{13-14}$. En este sentido, el módulo de elasticidad se relaciona con la calidad del hueso, es decir, cuanto menor es la densidad del hueso menor es el 
módulo. La cantidad de contacto hueso-implante es menor en el hueso menos denso y la resistencia del hueso se relaciona directamente con su densidad ósea. De hecho, cuanto más blando es el hueso maxilar más débiles son sus trabéculas óseas y su tasa de remodelación es más rápida, sobre todo, durante el proceso de carga inmediata. Además, el hueso cortical del maxilar superior es más delgado comparado con el mandibular ${ }^{17}$.

Ahora bien, la carga inmediata de los implantes no provoca necesariamente tensiones excesivas en el hueso. Se han realizado estudios que demuestran un elevado contacto directo entre el hueso y el implante cargado de forma inmediata sin alteración de la normal biología ósea con la presencia de los fenómenos de adhesión celular y aposición directa de hueso ${ }^{18-19}$. Lo que significa que la carga inmediata es compatible con la oseointegración y no provoca necesariamente una encapsulación fibrosa del implante ${ }^{17}$.

\section{ESTABIIDAD PRIMARIA}

Uno de los requisitos imprescindibles para conseguir y mantener la oseointegración es la estabilidad primaria de los implantes y la ausencia de movilidad durante la fase inicial de cicatrización ${ }^{20-21}$. Sin embargo los protocolos actuales de carga inmediata permiten la existencia de ciertos micromovimientos que parecen no afectar a una respuesta tisular ósea favorable ${ }^{22}$. La tendencia actual es aceptar que un cierto rango de micromovimientos no altera la oseointegración de forma absoluta y evita la encapsulación fibrosa de los implantes. Los estudios recientes demuestran que los micromovimientos por encima de 150 micrómetros son excesivos y por debajo de 50 micrómetros son tolerables ${ }^{13,18,22-23}$. Además, la tolerancia a los micromovimientos puede ser mayor entre los implantes con superficie rugosa ${ }^{18}$.

Durante la fase quirúrgica de la inserción de implantes en el maxilar superior para su carga inmediata es esencial realizar un fresado cuidadoso que configure un lecho implantario ideal para establecer una excelente estabilidad primaria. A veces, las características histológicas del hueso maxilar, sobre todo en el sector posterior no son favorables para la inserción de los implantes. En estos casos, la técnica quirúrgica con un menor fresado y la compactación del hueso mediante expansores, así como la utilización de implantes con un diseño apropiado (tipo de roscas para cada densidad de hueso, superficie rugosa, diámetro ancho) puede ayudar a obtener una mejor estabilidad primaria ${ }^{17}$. También la frecuencia de resonancia permite valorar la estabilidad primaria de los implantes y contraindicar la carga inmediata en aquellos que presentan valores menores de 55 ISQ ${ }^{24}$.

\section{IMPLANTES}

Los factores relacionados con los implantes son muy importantes en los protocolos de carga inmediata. El diseño, la superficie, el diámetro, la longitud y el número de implantes constituyen algunas características que configuran el éxito de esta terapéutica implantológica ${ }^{13,21,23,25}$.

El diseño de los implantes ha sido considerado un requisito esencial para el éxito del tratamiento con implantes. El diseño roscado de los implantes desarrolla una mayor retención mecánica así como una mayor capacidad para transmitir fuerzas compresivas. Además, el diseño roscado del implante disminuye la posibilidad de micromovimientos y mejora la estabilidad inicial que representa una condición imprescindible para el éxito de la carga inmediata ${ }^{13}$. El diseño del implante tiene un mayor impacto sobre el área de la superficie funcional que el tamaño del mismo. En este sentido, en algunos diseños un mayor diámetro del implante cilíndrico tiene menos área de superficie que un diámetro roscado. La forma de raíz del implante puede incrementar la fijación apical del mismo y mejorar la estabilidad primaria tan importante en aquellas zonas del maxilar superior donde la calidad del hueso es peor ${ }^{10,21}$. Además la profundidad, el número, y la orientación de las espiras roscadas puede afectar al área que va a resistir las fuerzas durante la carga inmediata. De hecho, la geometría de las espiras roscadas puede influir en la intensidad de la oseointegración precoz y en la interfase titaniohueso ${ }^{21}$.

Aunque existían hace ya algunos años se han vuelto a recuperar, recientemente, los diseños de implantes que incluyen el pilar protésico en una sola pieza (implante con unión protésica solidaria) (Figuras 5-6). Este diseño especialmente realizado para la carga inmediata tiene la ventaja de que va unido al implante en un solo cuerpo por lo que se evita la unión atornillada entre pilar e implante. Su principal desventaja es que debe ser insertado con cuidado para que el eje del implante se corresponda con la restauración protésica, por lo que en maxilares muy reabsorbidos con vestibulización de la cresta alveolar no están indicados. 
La superficie de los implantes puede afectar a la cantidad de contacto hueso implante y a la formación de hueso laminar. Cuando una superficie es modificada con una textura rugosa se observa un incremento importante en el contacto hueso-implante. La rugosidad del implante puede mejorar la cicatrización inicial y las condiciones de la carga inmediata ${ }^{26-27}$. Lo que significa que hacen falta fuerzas mayores para desinsertar a implantes con superficie rugosa comparados con los de superficie lisa ${ }^{13}$. La estabilidad primaria que proporciona la superficie rugosa del implante en comparación con la superficie lisa ha sido medida por frecuencia de resonancia demostrándose mayores valores de estabilización ${ }^{28}$. Además, las superficies lisas son menos exitosas sobre todo en aquellas condiciones óseas con bajo grado de densidad como ocurre frecuentemente, en las áreas posteriores del maxilar superior ${ }^{21}$. Los implantes con superficie rugosa (ej. grabados con doble ácido, chorreado de arena) están especialmente indicados en los casos de carga inmediata porque pueden prevenir la contracción del coágulo de fibrina sobre la superficie del implante devolviendo su capacidad osteoconductiva y favoreciendo la formación directa de hueso por las células osteogénicas ${ }^{12}$.

En los protocolos de carga inmediata en el maxilar superior edéntulo, el número de implantes puede influir al incrementar la superficie total funcional que recibe las fuerzas oclusales. En general, se recomienda un número mayor de implantes para realizar la carga inmediata con prótesis fija en el maxilar superior (Figura 6) que en la mandíbula lo que compensaría la menor densidad ósea y la dirección de las fuerzas vestibulares no favorables ${ }^{3,25}$.

El tamaño (longitud y diámetro) de los implantes puede también influir en los protocolos de carga inmediata. Por cada $3 \mathrm{~mm}$ de longitud, el área de superficie se incrementa un 20-30\%. Además, los fracasos implantarios son superiores cuando se utilizan implantes con una longitud menor de $10 \mathrm{~mm}$. Estas consideraciones son críticas en la carga inmediata del maxilar superior ya que la carga funcional ocurre antes del establecimiento de una conexión celular entre el hueso y la superficie del implante. En este sentido, la longitud del implante es todavía más relevante en los casos de mala calidad ósea. Además, la longitud del implante puede favorecer su fijación en la cortical superior lo que aumentaría la estabilidad primaria ${ }^{21}$.

El área funcional de cada implante puede incrementarse con el diámetro del mismo. Un mayor diámetro del implante se corresponde con un área incrementa- da de contacto hueso-implante. Como la función inmediata puede causar una pérdida precoz de hueso en la unión entre la superficie del implante y la cresta ósea alveolar, parece recomendable utilizar implantes más anchos para prevenir este problema. Además, se recomienda la inserción de implantes de diámetro mayor en los protocolos de carga inmediata en las zonas posteriores del maxilar superior para compensar la acción de las fuerzas oclusales sobre todo en los casos de baja densidad ósea ${ }^{21}$.

\section{CONCLUSIONES}

El presente trabajo demuestra la posibilidad de la inserción quirúrgica de implantes como primera fase en el tratamiento mediante la carga inmediata del maxilar edéntulo. Un diagnóstico preoperatorio y una correcta planificación mediante la realización de una técnica quirúrgica cuidadosa que valore la calidad y cantidad del hueso, así como la utilización de implantes con un diseño y superficie apropiados en número, longitud y diámetro permiten conseguir una estabilidad primaria para su posterior protocolo protésico.

\section{ABSTRACT}

Introduction. The aim of this paper was to present the surgical protocol of immediate loading of implants in the treatment of maxilla.

Immediate functional loading of dental implants for rehabilitation of maxilla constitute a comprehensive approach that include a preoperative assessment. Before surgery, systemic and oral documentation, and radiographic (orthopantomographs, dental scan) imaging were obtained. Antimicrobial prophylaxis was obtained with antibiotics (clavulanic acid and amoxicillin) and chlorhexidine. Local anesthesia was induced with articaine/epinephrine.

Surgery begans a bone crest incision or non invasive technique (non flap). One-piece implants may be inserted with a anterior-posterior distribution according a surgical guide. Appropiate surgical technique and a good design with rough surface of implant improve primary stability and osseointegration. Number and size of implants were selected according patient diagnosis and prosthetic assessment.

Conclusions. This paper indicate that surgical step of treatment of maxilla by immediate loading with 
osseointegrated implants may constitute a high successful treatment.

\section{KEYWORDS}

Dental implants, immediate loading, fixed prostheses, implant dentistry, maxilla.

\section{BIBLIOGRAFÍA}

1. Branemark PI, Zarb GA, Albrektsson T. Tissue-Integrated prostheses. Osseointegration in clinical dentistry. Berlin: Quintessence. 1987.

2. Schroeder A, Sutter F, Buser D, Krekeler G. Oral implantology. Basis, ITI hollow cylinder system. $2^{\mathrm{a}}$ ed. Nueva York: Thieme. 1996.

3. Chiapasco M. Early and immediate restorations and loading of implants in completely edentulous patients. Int J Oral Maxillofac Implants 2004; 19 (suppl):76-91.

4. Al-Omiri M, Hantash RA, Al-Wahadni A. Satisfaction with dental implants:a literature review. Implant Dent 2005; 14:399-408.

5. Chiapasco M, Gatti C, Rossi E, Haefliger W, Markwalder TH. Implant-retained mandibular overdentures with immediate loading:a retrospective multicenter study on 226 consecutive cases. Clin Oral Impl Res 1997;8:48-57.

6. Velasco E, Segura E, Linares D, Medel R, Poyato M. La carga inmediata de implantes transicionales en sobredentaduras mandibulares en adultos mayores. Av Perio Implant Oral 2004; 16:107-13.

7. Schnitman PA, Wohrle PS, Rubenstein JE. Immediate fixed provisional protheses supported by two-stage threated implants :metodology and results. J Oral Implantol 1990; 16:96-105.

8. Tarnow DP, Emtiaz S, Calssi A. Immediate loading of threated implants at stage 1 surgery in edentulous arches:Ten consecutive case reports with 1- to 5-year data. Int J Oral Maxillofac Implants 1997 ; 12 :319-24.

9. Velasco E, García A, Segura JJ, Medel R, López J. La carga funcional inmediata con implantes en pacientes edéntulos mandibulares. Técnica de Maló. Av Perio Implant Oral 2006; 18:
10. Degidi M, Piatelli A. Immediate functional and nonfunctional loading of dental implants: $A$ 2-to 60-months follo-up of 646 titanium implants. J Periodontol 2003; 74:225-41.

11. Degidi M, Piatelli A, Felice P, Carinci F. Immediate functional loading of edentulous maxilla:a 5-year retrospective study of 388 titanium implants. J Periodontol 2005; 76:1016-24.

12. Ibañez JC, Tahhan MJ, Zamar JA, Menendez AB, Juaneda AM, Zamar NJ, Monqaut JL. Immediate occlusal loading of double acid-etched surface titanium implants in 41 consecutive full-arch cases in the mandible and maxilla:6-to 74 -months results. J Periodontol 2005; 76:1972-81.

13. Gapski R, Wang HL, Mascareñas P, Lang NP. Critical review of immediate implant loading. Clin Oral Implant Res 2003; 14:515-27.

14. Lekholm U. Immediate/early loading of oral implants in compromised patients. Peridontology 2000 2003; 33: 194-203.

15. Jacobs R. Preoperative radiologic planning of implant surgery in compromised patients. Peridontology 2000 2003; 33:12-25.

16. Jiménez-López V. Carga o función inmediata en implantología. Aspectos quirúrgicos, protéticos, oclusales y de laboratorio. Barcelona:Quintessence. 2004.

17. Misch CE, Scortecci GM. Immediate load applications in implant dentistry. En:Misch CE. Dental implant prosthetics. San Luís:Elsevier Mosby. 2005. pag:531-67.

18. Meyer U, Wiesmann HP, Fillies T, Joos U. Early tissue reaction at the interface of immediately loaded dental implants. Int J Oral Maxillofac Implants 2003; 18:489-99.

19. Romanos GE, Testori T, Degidi M, Piatelli A. histologic and histomorphometric findings from retrieved, immediately occlusally loaded implants in Humans. J Periodontol 2005; 76:1823-32.

20. Buchs AU, Levine L, Moy P. Preliminary report of immediately loaded altiva natural tooth replacement dental implants. Clin Implant Dent Rel Res 2001; 3:97105.

21. Misch CE, Wang HL, Misch CM, Sharawy M, Lemons J, Judy KWM. Rationale for the application of immediate 
load in implant dentistry: Part II. Implant Dent 2004; 13: 310-21.

22. Brunski JB, Moccia AF, Pollock SR, Korostoff E, Tratchenberg DI. The influence of functional use of ensosseous dental implants on the tissue implant interface: I. Histological aspects. J Dent Res 1979;58:1953-69.

23. Szmukler-Moncler S, Piatelli A, Favero GA, Dubruille JH. Considerations preliminary to the application of early and immediate loading protocols in dental implantology. Clin Oral Impl Res 2000; 1 1:12-25.

24. Bischof M, Nedir R, Szmukler-Moncler S, Bernard JP, Samson J. Implant stability measurement of delayed and immediately loaded implants during healing. A clinical resonance-frequency analysis study with sandblasted and etched ITI implants. Clin Oral Implant Res 2004; 15:529-39.

25. Misch CE, Wang HL, Misch CM, Sharawy M, Lemons J, Judy KWM. Rationale for the application of immediate load in implant dentistry: Part I. Implant Dent 2004; 13: 207-17.
26. Butz F, Aita H, Wang CJ, Ogawa T. Harder and stiffer bone osseointegrated to roughened titanium. J Dent Res 2006; 85:560-5.

27. Shalabi MM, Gortemarker A, Van't Hof MA, Jansen JA, Creugers NHJ. Implant surface roughness and bone healing: a systematic review. J Dent Res 2006; 85: 496500.

28. Buser D, Schemk RK, Steinman S y cols. Influence of surface characteristics on bone integration of titanium implants. A histomorphometric study in miniature pigs. J Biomed Mater Res 1991;25:889-902.

\section{CORRESPONDENCIA}

Prof. Eugenio Velasco Ortega

Facultad de Odontología

Avicena, s/n

41009 Sevilla

Tfno: 954481132

e-mail: evelasco@us.es 